\title{
The Axiom of Choice in an Elementary Theory of Operations and Sets
}

\author{
John L. Bell \\ For Bill Demopoulos, colleague and friend
}

\section{Introduction: the Concepts of Operation and Function}

The terms "operation" and "function" are fundamental in the vocabulary of mathematics, and their meanings are closely related. Nevertheless those meanings are different. Operations, in the mathematical sense, were recognized and used millennia before the emergence of the idea of function. This can be seen above all from the fact that, in arithmetic, addition, multiplication, etc. are called operations, rather than functions. Given that (whole) numbers themselves arise from the fundamental operation of (intransitive) counting, the idea of an operation on numbers emerges almost automatically from the idea of number itself: it has the same kind of immediacy. Of significance also is the fact that numerical operations are homogeneous in that they transform numbers into numbers - like into like. Algebra arose through the recognition that the rules governing arithmetic operations could be extended to wider domains of entities presented as symbols, yet at the same time retaining the homogeneity of the arithmetic operations: for example, fractions by the Babylonians, quadratic expressions by the Hindus, and cubic expressions by the pre-Renaissance Italian mathematicians. Since algebraic operations act on symbols they are intensional in the sense that the result of applying such an operation to a symbol produces another symbol whose identity depends entirely on the identity of the first symbol, rather than on what that symbol may happen to denote - its "value". (By contrast, a procedure whose outputs depend only on the denotations or values of the inputs we shall deem extensional: see below.) For example, $2^{2}$ and 4 
both denote the same number; but while $\sqrt{2^{2}}$ and $\sqrt{4}$ both denote the number 2, as symbols they are entirely distinct. Of course, in a simple situation such as this the intensionality is easily "eliminated" by the application of rules of reduction, so allowing the derived symbols to be recognized as having the same value. But this is by no means always the case. A profound instance of the irreducible intensionality of algebraic operations in this sense - and a source of great puzzlement to the mathematicians of pre-Renaissance Italy - arose with the effort to solve irreducible cubic equations. As Cardano recognized, if Tartaglia's method is applied to the equation

$$
x^{3}-15 x+4=0,
$$

one obtains the solution

$$
x=\sqrt[3]{2+\sqrt{-121}}+\sqrt[3]{2-\sqrt{-121}} .
$$

But it is immediately clear that (1) is algebraically equivalent to the equation

$$
(x-4)\left(x^{2}+4 x+1\right)=0,
$$

which has the obvious solution

$$
x=4 .
$$

If we denote by $S$ the operation of solving a cubic equation, then $S$ applied directly to equation (1) yields solution $\left(1^{*}\right)$, while $S$ applied to the equivalent equation (2) yields the solution $\left(2^{*}\right)$, which is formally quite different from $\left(1^{*}\right)$. In this (an algebraically "irreducible" case) there are no rules of reduction enabling $\left(1^{*}\right)$ to be transformed into $\left(2^{*}\right)$. Thus $S$ is an example of an operation whose intensionality is essentially ineliminable - a truly "irreducible" case of intensionality.

Turning now to the concept of function, the idea can be traced to the analysis of motion undertaken by the mathematicians of the $17^{\text {th }}$ century, specifically, to the study of curves arising as paths of moving points. The term "function" in its mathematical sense was first introduced by Leibniz in 1673 to mean any quantity varying from point to point on a given curve (e.g. the length 
of the tangent), the curve itself being given by an equation. Leibniz later came to use "function" to signify a quantity, or quantities, whose values depend on a variable. The form or expression of, or the law governing such a dependence was, for a long time, taken to be an essential element in the idea of a function: for example, in 1667 James Gregory defined (what later become known as) a function to be any quantity obtained from other quantities by algebraic operations, or by "any other operation imaginable". Later John Bernoulli speaks of functions as quantities formed of variables and constants in an arbitrary manner: this last phrase was intended to allow for the inclusion of transcendental functions such as the trigonometric and exponential functions as well as algebraic. For Euler, who in 1734 introduced the standard notation $f(x)$ and whose Introductio of 1748 was the first work in which the function concept played a central role, the principal difference among functions arose from the mode of combination of the variables and constants comprising them. So for instance the transcendental functions, arising as infinite series, are distinguished from the algebraic functions by the fact that in the former the operations of the latter are iterated to infinity. $\mathrm{Up}$ to the end of the $18^{\text {th }}$ century the function concept may be said to have been conceived in a way similar to that of operation, that is, intensionally.

This state of affairs was to change in the 19th century, during which the concept of function underwent a vast expansion following the introduction of Fourier analysis. Even so, initially mathematicians still cleaved, by and large, to the view that a function must be representable by an analytic expression of some kind, despite the enlargement of the idea of analytic expression to include Fourier series. In 1837, however, Dirichlet took the decisive step of freeing the idea of a function from the mode of its expression, or "law" governing it by enunciating the definition that has since become standard, namely, that $y$ is a function of $x$ when to each value of $x$ (in a given interval) there corresponds a unique value of $y$. He emphasized that it is immaterial whether $y$ depends upon 
$x$ according to one law or more or whether that dependence can be expressed in terms of mathematical operations.

Dirichlet's definition ultimately led to the identification of functions within set theory as single-valued relations. Thus a function was conceived, in essence, as indicating an arbitrary dependence, or correspondence, between numbers ${ }^{1}$. This move enabled functions to be treated purely extensionally, in terms of its "range of values" or "graph". The intensionality ${ }^{2}$ associated with the old conception of function accordingly began to drop away.

Since mathematical operations also satisfied, in a formal sense, the same "uniqueness" condition as functions, these too were subsumed under the idea of single-valued relation. In this way an operation became identified as a special type of function, in fact, a function (and so, in particular, conceived extensionally) satisfying just the "homogeneity" condition that it associate like with like. Thus the essentially intensional character of operations also became obscured.

\section{The Axiom of Choice}

The extensional treatment of functions is implicit in Zermelo's 1904 formulation of what later became known as the Axiom of Choice:

Imagine that with every [nonempty] subset $M^{\prime}$ [of a given set $M$ ] there is associated an arbitrary element $m_{1}^{\prime}$ that occurs in $M^{\prime}$ itself... this yields a "covering" of the set $M$ [of nonempty subsets of $M$ ] by certain elements of $M$. The number of these coverings is equal to the product [of the cardinalities of] all subsets $M^{\prime}$ and is therefore certainly different from 0 .

\footnotetext{
${ }^{1}$ In the eleventh edition (1913) of the Encyclopedia Britannica we find the mathematical concept of function defined as a variable number, the value of which depends upon the values of one or more other variable numbers. This is essentially Dirichlet's definition.

${ }^{2}$ Nevertheless, it is of interest to observe that the old term fungible, itself derived from "function", is defined to mean "capable of mutual substitution" or "interchangeable"; that is, "having the same function". This would seem to indicate that the idea of function involved is extensional in the sense specified above.
} 
The Axiom of Choice is essentially the assertion that every nonempty set has at least one covering in this sense. From the passage above it seems clear that for Zermelo a "covering", obtained by associating an arbitrary element to each nonempty subset of a set, is essentially a function (a choice function) given extensionally. Indeed, not only does Zermelo omit to mention how such a function is to be defined, but the purely "numerical" (or combinatorial) justification he gives for the existence of coverings justifies the existence of the corresponding functions only when they are given in the extensional sense. From the assertion that "the number of these coverings is certainly different from 0 ", it does not follow that "there is a covering which can be described." And Zermelo was surely aware of this.

As is well known, Zermelo's use of the Axiom of Choice to prove the Well-Ordering Theorem gave rise to a storm of controversy. He was criticized chiefly on the grounds that in asserting the existence of a "covering" or choice function, Zermelo had provided no method of actually defining one. In their insistence that a (choice) function could not be considered to exist unless it was definable, Zermelo's critics were, unconsciously perhaps, cleaving to the old intensional conception of function. I do not think it would be overstepping the bounds of plausibility to maintain that Zermelo's critics were implicitly requiring choice functions to be presented as operations of some kind. In his 1908 formulation of the Axiom of Choice, Zermelo attempts to circumvent the whole issue of definability by replacing the notion of choice function by that of a transversal for a family of sets, but this move failed to silence his sterner crtitics.

Zermelo's 1904 formulation of the Axiom of Choice is equivalent to the assertion, for an arbitrary relation $R$ between sets $A, B$

(AC)

$$
\forall x \in A \exists y \in B R(x, y) \rightarrow(\exists f: A \rightarrow B) \forall x \in A R(x, f x) .
$$


Zermelo's critics would not accept the consequent of this implication unless an explicit description of the "choice" function $f: A \rightarrow B$ appearing there could be provided. So in effect they were insisting that $f$ be definable in some way. Let us be specific and construe "definable" as first-order definable (in the language of set theory), and write DAC for the version of $\mathrm{AC}$ in which the " $f$ " in the consequent is restricted to first-order definable functions. DAC is intended to provide a reasonable representation of what Zermelo's critics understood AC to mean. If that is granted, then Zermelo's critics were perfectly justified in questioning (what they understood by) the Axiom of Choice. For, as was shown in the 1960s, DAC can fail even when AC holds (in the usual set-theoretic sense), as follows from Feferman's construction of a model of ZFC in which the set of real numbers has no definable well-ordering. This is a striking and subtle instance of the (by now) familiar phenomenon of a function whose extension is guaranteed but which cannot be defined in a prescribed manner.

Now there is no reason to suppose that Zermelo's critics would have relaxed their insistence on the definability of the choice function $f$ even when the relation $R$ is single-valued. The Axiom of Choice for single-valued relations is usually called the Axiom of Unique Choice; it takes the form

(AUC)

$$
\forall x \in A \exists ! y \in B R(x, y) \rightarrow(\exists f: A \rightarrow B) \forall x \in A R(x, f x)
$$

In the usual set theories, functions are simply identified with single-valued relations: AUC is thereby reduced to a truism. (Indeed, AUC may be seen as a set-theoretic formulation of Dirichlet's definition of a function.) But if, in the spirit of Zermelo's critics, one requires in the consequent of AUC that the function $f$ be first-order definable - let us call the result DAUC - then the latter is certainly no truism, and in fact is no longer generally affirmable. For it is easy to show that DAUC holds in a model $\mathbf{M}$ of set theory if and only if $\mathbf{M}$ is 
pointwise definable, that is, if every element of $\mathbf{M}$ is first-order definable in $\mathbf{M}$. (An example of such a model is provided by the so-called minimal model.)

A mathematical context in which one would expect a "definable" version of AUC to hold is provided by recursive, or computable mathematics. For instance, let $\mathbf{N}$ denote the set of natural numbers and write CAUC for the assertion

$$
\forall x \in \mathbf{N} \exists ! y \in \mathbf{N} R(x, y) \rightarrow(\exists f: \mathbf{N} \rightarrow \mathbf{N})[\text { f recursive } \wedge \forall x \in \infty R(x, f x)] .
$$

CAUC amounts to what constructivists call the strong version of Church's thesis, namely the assertion that every total function $\mathbf{N} \rightarrow \mathbf{N}$ is recursive. While CAUC is compatible with set theory based on intuitionistic logic - it holds, for example, in the so-called effective topos - it is compatible with the usual set theory based on intuitionistic logic.

\section{A Proposal}

All this suggests the desirability of distinguishing the concepts of operation, function, and single-valued relation (i.e. set). Of course, in an important sense this step was taken some time ago by category theory, in which the ideas of operation and function are present in the vastly more general form of morphism or arrow, while the set concept, as such, has disappeared, or at least is present only in a residual sense as the notion of object, which itself can be defined in terms of morphism.

The theory presented here-an elementary theory of operations and sets, ETHOS for short - differs from the theory of categories in that sets, on the one hand, and operations and functions, on the other, are treated on a par: operations and functions are not "reduced" to sets of ordered pairs (as in set theory), but neither do sets vanish altogether (as in category theory). It will be formulated as a constructive theory based on intuititionistic logic ${ }^{3}$.

\footnotetext{
${ }^{3}$ For some related approaches, see Feferman [1975 ] and [1979 ].
} 
In ETHOS the idea of operation will be taken as primitive: it will be liberated from the "homogeneity" condition in that an operation will be allowed to have arbitrary, possibly differing, sets as domain and codomain. Most importantly, the concept of operation will be treated intensionally (as nature intended). The concept of function will play only a secondary role in our scheme. In fact, the notion will only arise in the form of (extensional) choice function on an indexed family of sets. ${ }^{4}$ Finally the concept of set will be entirely distinct from the concept of operation and subject just to rudimentary axioms, similar to those introduced in Bell [2008]. We shall see that ETHOS provides a natural framework for investigating the Axiom of Choice.

\section{The basic language and axioms of ETHOS}

Language. ETHOS is a theory presented in a two-sorted version $\mathbf{L}$ of the system of (intuitionistic) predicate logic with partial terms as formulated by Beeson [1985]. The equality relation $=$ in $\mathbf{L}$ as to be understood as intensional equality. In this system the rules for the formation of formulas and terms are as usual, but there is an additional rule:

- if $t$ is a term, then $t \Delta$ is an atomic formula (" $t$ is defined").

The propositional axioms and rules of inference are the usual intuitionistic ones. The quantifier axioms and rules are as follows:

$$
\begin{aligned}
& \quad \underline{A \rightarrow B} \quad \underline{B \rightarrow A} \\
& \exists x A \rightarrow B \quad \\
& \forall x A \wedge t \Delta \rightarrow A[t / x] \quad A[t / x] \wedge t \Delta \rightarrow \exists x A \\
& \text { - } \quad x=y \rightarrow y=x \\
& \text { - } \quad x \equiv t \wedge A(s) \rightarrow A(t), \text { where } s \equiv t \text { is } s \Delta \vee t \Delta \rightarrow s=t
\end{aligned}
$$

\footnotetext{
${ }^{4}$ More generally, a function can be conceived of as an operation defined on a set respecting a given equivalence relation on that set. Taking the equivalence relation is to represent the idea of "possessing the same value", an operation respecting such is then extensional in the sense that its outputs depend only on the "values" of its inputs.
} 
- $A\left(t_{1}, \ldots, t_{n}\right) \rightarrow t_{1} \Delta \wedge \ldots \wedge t_{n} \Delta \quad(A$ any atomic formula $)$

- $\quad c \Delta \quad(c$ any constant symbol)

- $\quad x \Delta \quad(x$ any variable $)$

L has two sorts: SET and OP. Terms of sort SET will be denoted by italic letters $x, y, z, t, u, v, \ldots, A, B, C, \ldots$ and terms of sort OP by greek letters $\varphi, \psi, \ldots, \Phi$, $\Psi, \ldots$. In addition to the equality symbol $=, \mathrm{L}$ also has the following constant, relation and function symbols 5 , each of which is assigned a signature as specified below:

- a relation symbol $\in$ of signature (SET, SET)

- a function symbols $\{\cdot, \cdot\}, \cdot \cup \cdot, \cdot x \cdot<\cdot>, \cdot$. each of signature((SET, SET), SET). We write $\{t\}$ for $\{t, t\}$.

- a constant symbol 0 of sort SET. We write 1 for $\{0\}$ and 2 for $\{0,1\}$.

- function symbols dom, cod, grph each of signature (OP, SET) : we write $\varphi: A \rightarrow B$ to indicate that $\operatorname{dom}(\varphi)$ is $A$ and $\operatorname{cod}(\varphi)$ is $B$. In that case, $\varphi$ is said to be between $A$ and $B$.

- a function symbol ap of signature ((OP, SET), SET): we write $\varphi(t)$ for $\operatorname{ap}(\varphi, t)$.

- a function symbol comp of signature ((OP, OP), OP): we write $\psi$ o $\varphi$ for $\operatorname{comp}(\varphi, \psi)$.

- function symbols res, cores both of signature ((OP, SET), OP): we write $\varphi \mid z$ for $\operatorname{res}(\varphi, Z)$ and $\left.\varphi\right|^{Z}$ for $\operatorname{cores}(\varphi, Z)$.

- a function symbol 1 of signature ((SET, SET), OP): we write $1 \times Y$ for $\mathfrak{i}(X, Y)$ and $\mathrm{iX}$ for $\mathrm{ixX}$.

- function symbols $\pi_{1}, \pi_{2}$ of signatures ((SET, SET), OP): when the sets $A, B$ are clear from the context, we write $\pi_{1}, \pi_{2}$ for $\pi_{1}(A, B), \pi_{2}(A, B)$.

\footnotetext{
${ }^{5}$ Here the term "function symbol" (which, strictly speaking, should be "operation symbol") is being used with its usual syntactic sense in formal systems.
} 
In addition $\mathbf{L}$ has certain abstraction terms: if $t(x)$ is any term, $A(x)$ any quantifier-free formula, both containing the free variable $x$, and $U$ any term of sort SET, then $\{t(x): A(x) \wedge x \in U\}$ is a term of sort SET.

Axioms. ETHOS has two groups of proper axioms SET axioms ${ }^{6}$.

- Empty set $\forall x \neg(x \in 0)$

- Unordered Pair $\forall x[x \in\{a, b\} \Leftrightarrow x=a \vee x=b]$

- Ordered Pair $\langle a, b\rangle=\langle c, d\rangle \Leftrightarrow a=c \wedge b=d$

- Binary Union $\forall x[x \in A \cup B \Leftrightarrow x \in A \vee x \in B]$

- Cartesian Product $\forall x[x \in A \times B \Leftrightarrow \exists y \in A \exists z \in B(x=\langle y, z\rangle)]$

- Primitive Replacement $\forall y[y \in\{t(x): x \in U \wedge A(x)\} \Leftrightarrow \exists x[x \in U \wedge A(x) \wedge$ $y=t(x)]], \quad$ where $A$ is any quantifier-free formula.

- $t \Delta$ where $t$ is any term of type SET containing only symbols of sort SET

- $\{t(x): A(x) \wedge x \in U\} \Delta$, where $A$ is any quantifier-free formula.

$\underline{\mathrm{OP} \text { axioms }}$

- $\varphi(x) \Delta \Leftrightarrow x \in \operatorname{dom}(\varphi)$

- $x \in \operatorname{dom}(\varphi) \Rightarrow \varphi(x) \in \operatorname{cod}(\varphi)$

- $(\psi \circ \varphi) \Delta \Leftrightarrow \operatorname{dom}(\psi)=\operatorname{cod}(\varphi)$

- $(\psi \circ \varphi) \Delta \Rightarrow \operatorname{dom}(\psi \circ \varphi)=\operatorname{dom}(\varphi) \wedge \operatorname{cod}(\psi \circ \varphi)=\operatorname{cod}(\varphi)$

- $\operatorname{dom}(\psi)=\operatorname{cod}(\varphi) \wedge x \in \operatorname{dom}(\varphi) \Rightarrow(\psi \circ \varphi)(x)=(\psi(\varphi(x))$

- $\forall u[u \in \operatorname{grph}(\varphi) \Leftrightarrow \exists x \in \operatorname{dom}(\varphi) . u=<x, \varphi(x)>]$

- $\quad \mathfrak{t}_{X Y} \Delta \Leftrightarrow X \subseteq Y^{7}$

- $X \subseteq Y \Rightarrow \operatorname{dom}\left(\mathrm{l}_{X Y}\right)=X \wedge \operatorname{cod}\left(\mathrm{l}_{X Y}\right)=Y \wedge \forall x \in X \mathrm{l}_{X Y}(x)=x$

\footnotetext{
${ }^{6}$ These axioms are related to those of the system RST - rudimentary set theory - introduced in Bell [2009 ]. See also Bell [2006].

${ }^{7}$ Here and in the sequel we employ standard set-theoretical terms such as "equivalence relation" and symbols and terms such as $\subseteq$ for inclusion.
} 
- $\operatorname{dom}\left(\pi_{1}(A, B)\right)=\operatorname{dom}\left(\pi_{2}(A, B)\right)=A \times B \wedge \operatorname{cod}\left(\pi_{1}(A, B)=A \wedge \operatorname{cod}\left(\pi_{2}(A, B)\right.\right.$ $=B \wedge \forall x \in A \forall y \in B\left[\pi_{1}(A, B)(<x, y>)=x \wedge \pi_{2}(A, B)(<x, y>)=y\right]$

- $\varphi \mid z \Delta \Leftrightarrow Z \subseteq \operatorname{dom}(\varphi)$

- $Z \subseteq \operatorname{dom}(\varphi) \Rightarrow \operatorname{dom}(\varphi \mid z)=Z \wedge \operatorname{cod}(\varphi \mid z)=\operatorname{cod}(\varphi) \wedge \forall x \in Z \varphi \mid z(x)=\varphi(x)$

- $\left.\varphi\right|^{Z} \Delta \Leftrightarrow Z \subseteq \operatorname{cod}(\varphi) \wedge \forall x \in \operatorname{dom}(\varphi) . \varphi(x) \in Z$

- $\left.\varphi\right|^{Z} \Delta \Leftrightarrow \operatorname{dom}\left(\left.\varphi\right|^{Z}\right)=\operatorname{dom}(\varphi) \wedge \operatorname{cod}\left(\left.\varphi\right|^{Z}\right)=\left.Z \wedge \forall x \in \operatorname{dom}(\varphi) \varphi\right|^{Z}(x)=\varphi(x)$

We shall write $A \approx B$ for $\forall x(x \in A \Leftrightarrow x \in B)$ and $\varphi \approx \psi$ for $\operatorname{dom}(\varphi)=$ $\operatorname{dom}(\psi) \wedge \operatorname{cod}(\varphi)=\operatorname{cod}(\psi) \wedge \forall x \in \operatorname{dom}(\varphi)(\varphi(x)=\psi(x)$. In both case the relation $\approx$ represents extensional equality.

From the OP axioms it is easily deduced that the collection $\mathbf{C}$ of sets and operations between them is a quasicategory, that is, satisfies the category axioms with o as composition, the $\imath_{X}^{\prime} s$ as identity arrows and $\approx$ as the identity relation between operations. In the quasicategorical sense, $\mathbf{C}$ has the terminal object 0 and the initial object $\{0\}$.

An operation $\varphi: A \rightarrow B$ is epi if $\forall y \in B \exists x \in A . y=\varphi(x)$.

An I-indexed family of subsets of a set $A$ is an operation $\varphi$ such that $\operatorname{dom}(\varphi)=I$ and $\forall i \in I \varphi(i) \subseteq A$. We shall usually write $X_{i}$ for $\varphi(i)$ and $\left\langle X_{i}: i \in I>\right.$ for $\varphi$.

\section{The Axiom of Choice in ETHOS 8}

ETHOS admits a number of natural formulations of the Axiom of Choice.

Let us call a binary relation (i.e. a set of ordered pairs) $R$ adequate on a set $X$ and write $\operatorname{Arel}(X, R)$ if $\forall x[x \in X \Leftrightarrow \exists y x R y]$; and operational on $X$, written

\footnotetext{
${ }^{8} \mathrm{~A}$ number of the ideas in this section were inspired by a reading of Martin-Löf [2006].
} 
$\operatorname{Orel}(X, R)$, if $\forall x[x \in X \Leftrightarrow \exists ! y x R y\}$. Then in ETHOS the usual Axiom of Choice takes the form

AC

$$
\operatorname{Arel}(X, R) \Rightarrow \exists \varphi[\operatorname{dom}(\varphi)=X \wedge \operatorname{grph}(\varphi) \subseteq R],
$$

and the Axiom of Unique Choice the form

AUC

$$
\operatorname{Orel}(X, R) \Rightarrow \exists \varphi[\operatorname{dom}(\varphi)=X \wedge \operatorname{grph}(\varphi) \approx R] .
$$

It is easy to see that AC implies AUC.

AUC enables operational relations to be replaced by authentic operations. This facility will be used principally to define indexed families of subsets. Thus, suppose given sets $I, A$ and a term $t(x)$ such that, for $i \in I, t(i) \subseteq A$. Then the relation $R=\{<i, t(i)>: i \in I\}$ is operational on $I$ and so AUC yields an operation $\varphi$ with domain $I$ for which $\operatorname{grph}(\varphi) \approx R$. It follows from this that, if we write $X_{i}$ for $t(i)$, then $X_{i}=\varphi(i)$ and so $\left\langle X_{i}: i \in I>\right.$ is an I-indexed family of subsets of $A$. Whenever AUC is assumed, we shall introduce indexed families of subsets in this way without further comment.

The Axiom of Choice can also be formulated in terms of indexed families of subsets. Let us define a choice operation on an I-indexed family of subsets $<X_{i}: i \in I>$ of a given set $A$ to be an operation $\varphi: I \rightarrow A$ for which

$$
\forall i \in I . \varphi(i) \in X_{i} .
$$

Now write $E$ for the equivalence relation on $I$ given by $i E j \leftrightarrow X_{i} \approx X_{j}$. An $E$ extensional choice operation on $\left\langle X_{i}: i \in I>\right.$ is called a choice function.

These definitions give rise to two further versions of the Axiom of Choice, namely:

ACO Any indexed family of nonempty ${ }^{9}$ subsets of a set admits a choice operation.

ACF Any indexed family of nonempty subsets of a set admits a choice function.

It is well-known that, in the usual intuitionistic set theory, the Axiom of Choice implies the Law of Excluded Middle (LEM). In ETHOS the situation is

\footnotetext{
${ }^{9}$ Here a set $X$ is said to be nonempty if $0 x . x \in X$.
} 
more involved: there AC only yields LEM if the Axiom of Extensionality (see below) is assumed for sets, and ACF only yields LEM in the presence of AUC. As for ACO, it does not yield LEM even given the Axiom of Extensionality, but it does so if one assumes both AUC and the existence of quotients of equivalence relations (the Axiom of Extensionality is not needed for the derivation).

We shall take the Law of Excluded Middle in the form:

LEM10 For any set $U, \forall x[x \in U \vee x \notin U]$.

It is to be observed that in this formulation LEM is asserted just for sets, not for operations. Notice that, in ETHOS, LEM is easily deducible from its "local" version which asserts that, for any sets $U \subseteq A, \forall x \in A[x \in U \vee x \notin U]$.

The Axiom of Extensionality for Sets is the sentence

Ext $\quad \forall U \forall V[U \approx V \Rightarrow U=V]$.

We now prove

Theorem 1. In ETHOS + Ext, AC implies LEM.

Proof. (b) Write 2 for $\{0,1\}$. Given a set $U$, define

$$
A=\{x \in 2: x=0 \vee y \in U\}, B=\{x \in 2: x=1 \vee y \in U\} .
$$

Then $A \subseteq 2$ and $B \subseteq 2$. Since $0 \in A$ and $1 \in B$, we have

$$
\forall x \in\{A, B\} \exists z \in 2 . z \in \mathcal{X},
$$

and so $\mathrm{AC}$ applied to the relation

$$
R=\{\langle x, z\rangle \in\{A, B\} \times 2: z \in x\}
$$

yields an operation $\varphi:\{A, B\} \rightarrow 2$ for which $\forall x \in\{A, B\} . \varphi(x) \in \mathcal{x}$. It follows that $\varphi(A) \in A \wedge \varphi(B) \in B$, so that

$$
[\varphi(A)=0 \vee y \in U] \wedge[\varphi(B)=1 \vee y \in U] .
$$

Applying the distributive law, we then get

$$
y \in U \vee[\varphi(A)=0 \wedge \varphi(B)=1]
$$

\footnotetext{
${ }^{10}$ This is the principle of detachability introduced in Bell [2009].
} 
whence

$$
y \in U \vee \varphi(A) \neq \varphi(B) .
$$

Now clearly $y \in U \Rightarrow A \approx B$ (both then being $\approx 2$ ), and so, assuming Ext, $y \in U \Rightarrow A=B$, from which we deduce $y \in U \Rightarrow \varphi(A)=\varphi(B)$, whence

$$
\varphi(A) \neq \varphi(B) \Rightarrow y \notin U \text {. }
$$

(1) and (2) yield

$$
y \in U \vee y \notin U,
$$

i.e. LEM.

Notice that Ext was needed to obtain (2); in its absence the argument does not go through.

Theorem 2. In ETHOS + AUC, LEM is deducible from ACF.

Proof. We derive LEM in its "local" form. Given sets $U \subseteq A$, define $I=A \times 2$ and for each $i=\left\langle a, k>\in I\right.$ let $X_{i}=\{n \in 2: n=k \vee a \in U\}$. Then $\left\langle X_{i}: i \in I>\right.$ is an $I-$ indexed family of nonempty subsets of 2, so ACF yields a choice function on $<X_{i}: i \in I$, i.e. an operation $\varphi: I \rightarrow 2$ such that

$$
\forall i \in I \varphi(i) \in X_{i} \wedge \forall i j \in I\left[X_{i} \approx X_{j} \Rightarrow \varphi(i)=\varphi(j)\right] .
$$

Accordingly for every $a \in A$,

$$
\varphi(<a, 0>) \in X_{<a, 0>} \wedge \varphi(<a, 1>) \in X_{<a, 1>,}
$$

in other terms,

$$
[\varphi(<a, 0>)=0 \vee a \in U] \wedge[\varphi(<a, 1>)=1 \vee a \in U] .
$$

Therefore

$$
a \in U \vee[\varphi(<a, 0>)=0 \wedge \varphi(<a, 1>)=1],
$$

whence

$$
a \in U \vee[\varphi(<a, 0>) \neq \varphi(<a, 1>)],
$$


Now clearly $a \in U \Rightarrow X_{<a, 0>} \approx X_{<a, 1>}$ (since both are then $\approx 2$ ), so that, since $\varphi$ is a choice function,

$$
a \in U \Rightarrow \varphi(<a, 0>)=\varphi(<a, 1>),
$$

whence

$$
\varphi(<a, 0>) \neq \varphi(<a, 1>) \Rightarrow a \notin U .
$$

This, together with (1), gives

$$
a \in U \vee a \notin U,
$$

whence LEM.

Observe again that the argument requires that $\varphi$ be a choice function rather than merely a choice operation as specified in ACO. The latter is considerably weaker than ACF and not strong enough on its own to yield LEM. We look finally, then, into the problem of specifying additional assumptions sufficient to enable ACO to become equivalent to ACF and hence to yield LEM.

We introduce the following principles:

Quotients. For any equivalence relation $\sim$ on a set $I$, there is a set $I^{*}$ and an epi $\varepsilon: I \rightarrow I^{*}$ such that $i \sim j \Leftrightarrow \varepsilon(i)=\varepsilon(j)$.

Representatives. For any equivalence relation $\sim$ on a set $I$, there is an operation $\varphi: I \rightarrow I$ such that $\varphi(i) \sim i$ and $i \sim j \Rightarrow \varphi(i)=\varphi(j)$.

Representatives asserts that representatives can be selected from the equivalence classes of any equivalence relation. In ETHOS, Quotients is deducible from Representatives. For, given an equivalence relation $\sim$ on a set $I$, let $\varphi: I \rightarrow I$ satisfy $\varphi(i) \sim i$ and $i \sim j \Rightarrow \varphi(i)=\varphi(j)$, as provided by Representatives. Defining $I^{*}=\{\varphi(i): i \in I\}$, and $\varepsilon=\left.\varphi\right|^{I^{*}}$, it is easy to verify that $\varepsilon: I \rightarrow I^{*}$ is epi and satisfies $i \sim j \Leftrightarrow \varepsilon(i)=\varepsilon(j)$.

Theorem 3. In ETHOS + AUC,

$$
\text { ACO + Quotients } \Rightarrow \text { Representatives. }
$$


Proof. Given a an equivalence relation $\sim$ on a set $I$, use Quotients to get an epi $\varepsilon: I \rightarrow I^{*}$ such that $i \sim j \Leftrightarrow \varepsilon(i)=\varepsilon(j)$. For each $u \in I^{*}$ define $X_{u}=\{i \in I: \varepsilon(i)=u\}$. Then $\left\langle X_{u}: u \in I^{*}\right\rangle$ is an $I^{*}$-indexed family of nonempty subsets of $I$ and so ACO gives $\psi: I^{*} \rightarrow I$ such that $\forall u \in I^{*} . \psi(u) \in X_{u}$, i. e.,

$$
\forall u \in I^{*} . \varepsilon(\psi(u))=u .
$$

Now define $\varphi: I \rightarrow I$ by $\varphi=\psi$ o $\varepsilon$. Then

$$
\varepsilon(\varphi(i))=\varepsilon(\psi(\varepsilon(i)))=\varepsilon(i),
$$

whence $\varphi(i) \sim i$. And

$$
i \sim j \Rightarrow \varepsilon(i)=\varepsilon(j) \Rightarrow \varphi(i)=\psi(\varepsilon(i))=\psi(\varepsilon(j))=\varphi(j) .
$$

Representatives follows.

Theorem 4. In ETHOS + AUC, Representatives and ACF are equivalent.

Proof. Representatives $\Rightarrow$ ACF. Let $<X_{i}: i \in I>$ be an indexed family of nonempty subsets of a set $A$. Defining $X_{i}^{\prime}=\{i\} \times X_{i},\left\langle X_{i}^{\prime}: i \in I>\right.$ is then an indexed family of subsets of the set $U=\left\{\left\langle i, x>\in I \times A: x \in X_{i}\right\}\right.$. Define the equivalence relation on $U$ by

$$
<i, x>\sim<j, y>\Leftrightarrow X_{i} \approx X_{j} .
$$

Representatives then gives $\varphi: U \rightarrow U$ satisfying

(1) $\varphi(<i, x>) \sim<i, x>$

(2) $<i, x>\sim<j, y>\Rightarrow \varphi(<i, x>)=\varphi(<j, y>)$.

Writing $\varphi_{1}=\pi_{1} \circ \varphi, \varphi_{2}=\pi_{2} \circ \varphi,(1)$ and (2) yield respectively

(3) $\varphi_{1}(<i, x>) \in X_{i}$

(4) $X_{i} \approx X_{j} \wedge x \in X_{i} \wedge y \in X_{j} \Rightarrow \varphi_{1}(<i, x>)=\varphi_{1}(<j, y>)$

Now define

$$
R=\left\{<\pi_{1}(u), \varphi_{1}(u)>: u \in I \times A \wedge \pi_{2}(u) \in X_{\pi_{1}(u)}\right\} .
$$

If $\pi_{1}(u)=\pi_{1}(v)$ with $\pi_{2}(u) \in X_{\pi_{1}(u)}$ and $\pi_{2}(v) \in X_{\pi_{1}(v)}$, then $X_{\pi_{1}(u)}=X_{\pi_{1}(v)}$ and $\varphi_{1}(u)$ $=\varphi_{1}(v)$ from (4). It follows that $R$ is operational. Accordingly by AUC there is an 
operation $\psi: I \rightarrow A$ for which $\operatorname{grph}(\psi) \approx R$. From the fact that $\left\langle i, \varphi_{1}(<i, x>)>\in R\right.$ for any $x \in X_{i}$, it follows easily that

$$
\forall x \in X_{i}\left[\psi(i)=\varphi_{1}(<i, x>)\right] .
$$

We claim that $\psi$ is a choice function on $\left\langle X_{i}: i \in I\right\rangle$. First, $\psi$ is a choice operation since, for any $x \in X_{i}, \psi(i)=\varphi_{1}(<i, x>) \in X_{i}$ by (3) and (5). To show that $\psi$ is a choice function, suppose that $X_{1} \approx X_{j}$, and choose any $x \in X_{i}$. Then $x \in X_{j}$ and so by (4) and (5)

$$
\psi(i)=\varphi_{1}(<i, x>)=\varphi_{1}(<j, x>)=\psi(j) .
$$

ACF $\Rightarrow$ Representatives. Given an equivalence relation $\sim$ on a set $I$, let $X_{i}=\{j \in I: i \sim j\}$. Then $\left\langle X_{i:} i \in I>\right.$ is an $I$-indexed family of nonempty subsets of $I$ and so by ACF there is a choice function on $\left\langle X_{i}: i \in I\right\rangle$. This is an operation $\varphi: I \rightarrow I$ such that (a) $\forall i \in I . \varphi(i) \in X_{i}$, i.e. $\varphi(i) \sim i$; and (b) $i \sim j \Rightarrow X_{i} \approx X_{j} \Rightarrow$ $\varphi(i)=\varphi(j)$. Representatives follows.

\section{Corollary. In ETHOS +AUC,}

\section{ACO + Quotients $\Leftrightarrow$ Representatives $\Leftrightarrow$ ACF.}

In effect (assuming AUC), Quotients is what is needed to convert choice operations into choice functions, and so to allow ACO to become equivalent to ACF.

Remark. In constructive type theories the version ACO of the Axiom of Choice is actually provable. Since LEM does not hold in these theories, it follows that neither ACF nor Quotients is provable there.

\section{References}

Beeson, M. [1985] Foundations of Constructive Mathematics. Berlin: Springer-Verlag Bell, J. L. [2009]. The Axiom of Choice. College Press. 
Bell, J. L. [2008]. The axiom of choice and the law of excluded middle in weak set theories", Mathematical Logic Quarterly, 54, no. 2.

Feferman, S. [1975]. A language and axioms for explicit mathematics. In Algebra and Logic, Springer Lecture Notes in Mathematics, 450, 87-139.

Feferman, S. [1979]. Constructive theories of functions and classes. In: Logic Colloquium '78, North-Holland Publ. Co., 159-224.

Martin-Löf, P. [2006]. 100 years of Zermelo's axiom of choice: what was the problem with it? Computer J. 49, $345-350$. 